\title{
Matroid Enumeration for Incidence Geometry
}

\author{
Yoshitake Matsumoto • Sonoko Moriyama • \\ Hiroshi Imai · David Bremner
}

Received: 30 August 2009 / Revised: 25 October 2011 / Accepted: 4 November 2011 /

Published online: 30 November 2011

(C) Springer Science+Business Media, LLC 2011

\begin{abstract}
Matroids are combinatorial abstractions for point configurations and hyperplane arrangements, which are fundamental objects in discrete geometry. Matroids merely encode incidence information of geometric configurations such as collinearity or coplanarity, but they are still enough to describe many problems in discrete geometry, which are called incidence problems. We investigate two kinds of incidence problem, the points-lines-planes conjecture and the so-called Sylvester-Gallai type problems derived from the Sylvester-Gallai theorem, by developing a new algorithm for the enumeration of non-isomorphic matroids. We confirm the conjectures of Welsh-Seymour on $\leq 11$ points in $\mathbb{R}^{3}$ and that of Motzkin on $\leq 12$ lines in $\mathbb{R}^{2}$, extending previous results. With respect to matroids, this algorithm succeeds to enumerate a complete list of the isomorph-free rank 4 matroids on 10 elements. When geometric configurations corresponding to specific matroids are of interest in some incidence problems, they should be analyzed on oriented matroids. Using an encoding of oriented matroid axioms as a boolean satisfiability (SAT) problem, we also enumerate oriented matroids from the matroids of rank 3 on $n \leq 12$ elements and rank 4 on $n \leq 9$ elements. We further list several new minimal non-orientable matroids.
\end{abstract}

\footnotetext{
Y. Matsumoto $\cdot$ H. Imai

Graduate School of Information Science and Technology, University of Tokyo, Tokyo, Japan

Y. Matsumoto

e-mail: ymatsu@is.s.u-tokyo.ac.jp

H. Imai

e-mail: imai@is.s.u-tokyo.ac.jp

S. Moriyama (凶)

Graduate School of Information Sciences, Tohoku University, Sendai, Japan

e-mail: moriso@dais.is.tohoku.ac.jp

D. Bremner

Faculty of Computer Science, Univesity of New Brunswick, Fredericton, Canada

e-mail: bremner@unb.ca
} 
Keywords Incidence geometry $\cdot$ Matroids $\cdot$ Oriented matroids

\section{Introduction}

A central topic in discrete geometry is the combinatorial structure of point configurations and hyperplane arrangements. It is often difficult to characterize the possible combinatorial structures of these geometric models, hence one strategy is to consider combinatorial abstractions such as matroids and oriented matroids, where particularly computational approaches tend to be much simplified. In this paper we focus on matroids, which model only incidence properties. This introduction, and the remainder of the paper are divided into three main parts. First we discuss some new results in incidence geometry obtained via an expanded catalog of matroids. Second we explain the algorithm used to generate this catalog. Third, we consider how to generate oriented matroids from matroids.

\subsection{Incidence Problems in Discrete Geometry}

Matroids and oriented matroids are important as combinatorial abstractions for point configurations and hyperplane arrangements, which are fundamental objects in discrete geometry. While oriented matroids describe relative positions of objects, matroids merely encode incidence information such as collinearity or coplanarity. If two of these geometric configurations determine the same matroid, we say they are $M$ equivalent. Similarly, if they determine the same oriented matroid, we say they are OM-equivalent. We mention that OM-equivalency is the usual definition of equivalency for these geometric configurations, although we use the term OM-equivalency for precision.

Because no direct method is known for enumeration of combinatorial structures of point configurations or hyperplane arrangements, the generation of these combinatorial abstractions is a promising approach. Note that there exist matroids and oriented matroids which cannot be realized by any point configuration or any hyperplane arrangement. At least for oriented matroids, several studies have been made on practical methods for the realizability problem which work satisfactorily for small instances [1, $2,21]$. On the other hand, in some cases, there is no need to identify the realizable matroids or oriented matroids. For instance, if a particular conjecture for point configurations or hyperplane arrangements is true for all matroids or oriented matroids, it is also true for realizable ones, which correspond to actual geometric configurations.

Although matroids encode only part of the combinatorial properties contained in oriented matroids, they are still enough to describe many problems in discrete geometry. We call such problems incidence problems. For incidence problems, enumeration of matroids is more useful than that of oriented matroids because it can offer information on configurations of a larger number of objects. Furthermore, when geometric configurations corresponding to specific matroids are of interest, we can investigate their structures at the OM-equivalency level by enumerating oriented matroids from the matroids.

In Sect. 2.1, we study the points-lines-planes conjecture by Welsh [40], an inequality relating the number of points to the number of lines and planes spanned by 
those points. This inequality is also conjectured to hold for (not necessarily realizable) matroids. We confirm this conjecture for matroids of rank 4 (and thus for point configurations in $\mathbb{R}^{3}$ ) on $n \leq 11$ elements, by restricting our search to extensions of a small subset of the rank 4 matroids on ten elements.

In Sect. 2.2, we analyze so-called Sylvester-Gallai type problems. This is a collection of problems derived from the Sylvester-Gallai theorem [15, 16, 30, 39]: any point configuration has an ordinary line, which passes exactly two of the given points, unless all points are on the same line. A lower bound $\lfloor n / 2\rfloor$ on the number of ordinary lines was conjectured by Motzkin [13, 24, 31]. We determine all extremal instances on $n \leq 12$ points in $\mathbb{R}^{2}$ (confirming the conjecture of Motzkin), and for $n \leq 10$ points in $\mathbb{R}^{4}$.

The advantage of the matroid-based approach is shown by the fact that our results in Sect. 2 are on configurations of a larger number of objects compared other computational approaches. In the next section, we discuss a new algorithm for the enumeration of non-isomorphic matroids.

\subsection{Enumeration of Matroids}

For many extremal problems in discrete geometry, the only known computational approach is one based on enumeration of possible (abstract) configurations. Even when the complete search space is not visited, more efficient techniques for enumeration can be useful as part of some other algorithm. In this paper we focus on incidence problems, where matroids are a good model. We are able to find the new results discussed above thanks to an improved algorithm for the enumeration of matroids.

The enumeration of matroids has been studied as part of the effort to classify matroids with small rank and ground set. Mainly isomorph-free enumeration has been considered because many properties of matroids are invariant under isomorphism. Since Blackburn, Crapo and Higgs [5] enumerated all isomorph-free simple matroids on $n \leq 8$ elements in 1973, many studies have been done to extend the classification of matroids. For rank 3 simple matroids, Betten and Betten [3] reached $n \leq 12$ elements by exploiting special properties of them. Recently Mayhew and Royle [28] achieved the enumeration of all isomorph-free matroids of any rank on $n \leq 9$ elements. For a special class of matroids called paving matroids, Mayhew and Royle determined all isomorph-free (paving) matroids of rank 4 on ten elements by using the correspondence between paving matroids and independent sets in a Johnson graph and its extensions. However, non-paving matroids of rank 4 on ten elements were not enumerated.

In this paper we propose a new algorithm for enumeration of matroids and complete the enumeration of matroids of rank 4 on ten elements with this algorithm.

Theorem 1 There are 4,886,380,924 isomorph-free matroids of rank 4 on ten elements.

Table 1 summarizes the number of matroids of rank $r$ on $n$ elements determined by our enumeration. The case $(r, n)=(4,10)$ and its dual $(r, n)=(6,10)$ are essentially new results by our enumeration. Other cases are confirmation of the previous studies and their dual or extension to non-simple ones. 
Table 1 Number of non-isomorphic matroids

\begin{tabular}{|c|c|c|c|c|c|c|c|c|c|c|c|c|c|}
\hline$r \backslash n$ & 0 & 1 & 2 & 3 & 4 & 5 & 6 & 7 & 8 & 9 & 10 & 11 & 12 \\
\hline 0 & 1 & 1 & 1 & 1 & 1 & 1 & 1 & 1 & 1 & 1 & 1 & 1 & 1 \\
\hline 1 & & 1 & 2 & 3 & 4 & 5 & 6 & 7 & 8 & 9 & 10 & 11 & 12 \\
\hline 2 & & & 1 & 3 & 7 & 13 & 23 & 37 & 58 & 87 & 128 & 183 & 259 \\
\hline 3 & & & & 1 & 4 & 13 & 38 & 108 & 325 & 1275 & 10037 & 298491 & 31899134 \\
\hline 4 & & & & & 1 & 5 & 23 & 108 & 940 & 190214 & 4886380924 & * & * \\
\hline 5 & & & & & & 1 & 6 & 37 & 325 & 190214 & $*$ & $*$ & $*$ \\
\hline 6 & & & & & & & 1 & 7 & 58 & 1275 & 4886380924 & $*$ & * \\
\hline 7 & & & & & & & & 1 & 8 & 87 & 10037 & $*$ & $*$ \\
\hline 8 & & & & & & & & & 1 & 9 & 128 & 298491 & * \\
\hline 9 & & & & & & & & & & 1 & 10 & 183 & 31899134 \\
\hline 10 & & & & & & & & & & & 1 & 11 & 259 \\
\hline 11 & & & & & & & & & & & & 1 & 12 \\
\hline 12 & & & & & & & & & & & & & 1 \\
\hline
\end{tabular}

Notes: $r$ : rank $n$ : size of the ground set

*Enumeration not completed

Here we mention that Mayhew and Royle's experiment using random generation suggests there are at least $2.5 \times 10^{12}$ matroids of rank 5 on ten elements. Enumeration of all matroids on ten elements is an attractive task but it seems difficult under the computational power of current computers. In that sense, our results may be considered as reaching the current limit.

\subsection{From Matroids to Oriented Matroids}

We use our results on the matroid enumeration to improve enumerative study of oriented matroids. An oriented matroid can be described roughly as a matroid in which every basis is signed. The oriented matroid is called an orientation of the underlying matroid. On the other hand, not every matroid has an orientation. Matroids with at least one orientation (i.e., which are underlying matroids of some oriented matroid), are called orientable matroids.

In general, orientable matroids have many orientations. Therefore the number of oriented matroids is much larger than that of matroids. Oriented matroids of rank $r$ on $n$ elements are determined for $r=3, n \leq 10$ and $r=4, n \leq 8$ by Finschi and Fukuda [18-20]. In the case of uniform oriented matroids, those of rank 3 on $n \leq 11$ elements were determined by Aichholzer and Krasser [2]. Compared with the enumeration of matroids, these enumerations of oriented matroids cover those with relatively small ground sets. This difference in coverage arises from the much larger number of oriented matroids with given parameters. For example, in the case of rank 3 and the ground set with ten elements, there are 95,052,532 simple oriented matroids while there are only 5249 simple matroids. The relative completeness of the classification of (unoriented) matroids motivates us to utilize it in computational analysis of oriented matroids with larger ground sets. We are thus concerned with two further 
Table 2 Results on orientability of matroids

\begin{tabular}{|c|c|c|c|c|c|c|c|c|c|c|}
\hline \multirow{2}{*}{$\begin{array}{l}\text { Rank } r \\
\text { Size } n\end{array}$} & \multicolumn{6}{|l|}{3} & \multicolumn{4}{|l|}{4} \\
\hline & 7 & 8 & 9 & 10 & 11 & 12 & 7 & 8 & 9 & $10^{*}$ \\
\hline \# simple matroids & 23 & 68 & 383 & 5249 & 232928 & 28872972 & 49 & 617 & 185981 & 1000000 \\
\hline \# orientable & 22 & 65 & 365 & 5048 & 223515 & 26873051 & 48 & 583 & 173697 & 696710 \\
\hline \# non-orientable & 1 & 3 & 18 & 201 & 9413 & 1999921 & 1 & 34 & 12284 & 303290 \\
\hline Ratio of non-orientable & 0.043 & 0.044 & 0.046 & 0.038 & 0.040 & 0.069 & 0.020 & 0.055 & 0.066 & 0.303 \\
\hline \# minimal non-orientable & 1 & 1 & 2 & 23 & 1458 & 397240 & 1 & 10 & 8481 & \\
\hline
\end{tabular}

* For the case with $r=4$ and $n=10$, we test a random sample of 1000000 matroids

problems: testing orientability and generating orientations of matroids. Orientability of matroids is a fundamental problem in understanding the gap between the model of matroids and that of oriented matroids. Generating orientations also serves as a new oriented matroid generation method. This local generation method is useful in cases where we are interested in oriented matroids with specific underlying matroids. Such cases are common because there are many properties of an oriented matroid determined by its underlying matroid.

We propose a method for testing orientability of matroids based on known [6, 37] ideas for oriented matroid generation using the Boolean Satisfiability (SAT) problem. Although testing orientability of matroids is known to be NP-complete [35], experimentally our method achieves satisfactory results. The motivation for this approach is the fact that although SAT is also NP-complete, practical heuristics [11, 12] and fast implementations [14] are known. Using our method we determine orientability of matroids with $r=3, n \leq 12$ and $r=4, n \leq 9$. This classification-more extensive than that obtained from existing enumeration of oriented matroids-is summarized in Table 2.

It has been conjectured that asymptotically most matroids are non-orientable [4, p. 279]. If this conjecture is true, it is expected that the ratio of non-orientable matroids is higher among matroids on larger ground sets. In a case of rank 3, the ratio of non-orientable matroids does not show clear increasing tendency according to $n$ for $n \leq 11$, but the case $n=12$ may be a start of growth. In the rank 4 case, we can observe a clearer increasing tendency. This behavior is consistent with the conjecture.

Non-orientable matroids have been studied because show the gap between matroids and oriented matroids. We list minimal non-orientable matroids following the known examples such as the Fano matroid and the MacLane matroid. We also investigate the strength of existing certificates of non-orientability [4, p. 273] by classifying non-orientable matroids according to them.

We can apply the previously noted oriented matroid generation methods using SAT $[6,37]$ to find all orientations of an orientable matroid. This works well if a matroid does not have too many orientations. We present a computational experiment that suggests while uniform matroids have many orientations, most other matroids, especially highly degenerate ones, have many fewer orientations. The result suggests generating all orientations via SAT is a practical approach for many inputs. 


\section{Incidence Problems in Discrete Geometry}

In this section, we present new results in discrete geometry obtained from enumeration of matroids (Sect. 3) and analysis of their orientations (Sect. 4). Section 2.1 confirms the points-lines-planes conjecture for $n \leq 11$ points. In this case, the derived bounds for matroids directly imply those for the geometric case. Section 2.2 analyzes the number of ordinary lines in point configurations in $\mathbb{R}^{2}$ and the number of ordinary planes in point configurations in $\mathbb{R}^{3}$. We also obtain results for the more general (dual) problem of simple crossings in pseudohyperplane arrangements.

Before proceeding to these results, we discuss the matroid setting in which we obtain them. We start by describing some of main ideas and notation we need from the matroid literature. For more background on matroids, we refer to the book by Oxley [33]. There are many equivalent ways to define matroids. In this paper, we regard a matroid as an ordered pair $(E, r)$ where $E$ is a ground set and $r: 2^{E} \rightarrow \mathbb{Z}$ is a rank function satisfying $0 \leq r(X) \leq|X|, X \subset Y \Rightarrow r(X) \leq r(Y)$, and $r(X \cup Y)+$ $r(X \cap Y) \leq r(X)+r(Y)$. The last property is called submodularity. The rank of a matroid $M=(E, r)$ is $r(E)$. The closure of $X$, denoted by $\operatorname{cl}(X)$, is the set of all $e \in E$ such that $r(X \cup\{e\})=r(X)$. $X$ is a flat if $\operatorname{cl}(X)=X$.

Two matroids $M_{1}=\left(E_{1}, r_{1}\right)$ and $M_{2}=\left(E_{2}, r_{2}\right)$ are isomorphic if there is a bijection $\psi$ from $E_{1}$ to $E_{2}$ such that $r_{1}(X)=r_{2}(\psi(X))$ for all $X \subseteq E_{1}$. The set of isomorphism classes of rank $r$ matroids on $n$ elements is denoted by $\operatorname{IC}(n, r)$.

From the definition, a flat of a matroid is a maximal set of elements of a given rank. Here we give geometric interpretations of this notion for point configurations and arrangements of hyperplanes. First, consider a point configuration $\mathcal{P}$ in $\mathbb{R}^{d}$. It determines a matroid of rank $d+1$ in a following manner: a subset $S$ of these points is a flat if there is an affine subspace passing through all points in $S$ but no other. The set of such flats determines the matroid. See Fig. 1 (left) for an example of the set of flats defined by a point configuration. It should be noted here that the term flat is also used to mean an affine subspace of $\mathbb{R}^{d}$. If a flat $S$ lies in a $k$-dimensional affine subspace but not in a $(k-1)$-dimensional one, $S$ is a flat of rank $k+1$ (the empty set is a flat of rank 0). By analogy with the geometric case, flats of a matroid of rank 1, 2, and 3 of a matroid are called points, lines, and planes. Next, consider a hyperplane arrangement. Here we consider one not in $\mathbb{R}^{d}$ but in a real projective space $\mathbb{P}^{d}$ where parallel hyperplanes intersect at infinity. This is because we want to avoid the hassle of treating parallel hyperplanes as special. We call a maximal set $S$ of these hyperplanes a flat of rank $k$ if its intersection is a $(d-k)$-dimensional affine subspace. Again the set of flats determines the matroid. The line arrangement in the right of Fig. 1 determines the same matroid as a point configuration in the left of Fig. 1 .

On the other hand, there is not always a point configuration or a hyperplane arrangement which determines a given matroid $M$. Let $A$ be a matrix $A$ on a field $F$, and $c(A)$ the set of columns of $A$. $A$ determines a matroid $M=(E, r)$ where $E=c(A)$ and $r(X)$ is the linear rank of $X \subseteq c(A) . M$ is called the vector matroid of $A$. If $M$ is isomorphic to the vector matroid of a matrix $A$ over a field $F, M$ is said to be representable over $F$ or $F$-representable. $A$ is called a representation for $M$ over $F$. Given a matroid $M$, there exists a matrix $A$ which is a representation for 


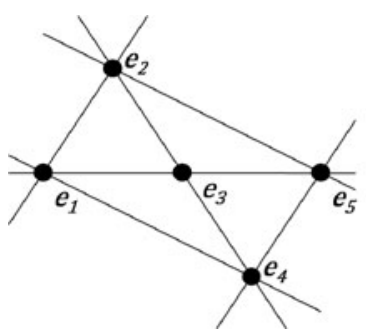

\begin{tabular}{|l|l|}
\hline rank & flat \\
\hline 0 & base \\
\hline 1 & $\left\{e_{1}\right\},\left\{e_{2}\right\},\left\{e_{3}\right\}$, \\
& $\left\{e_{4}\right\},\left\{e_{5}\right\}$ \\
\hline 2 & $\left\{e_{1}, e_{3}, e_{5}\right\}$, \\
& $\left\{e_{2}, e_{3}, e_{4}\right\}$, \\
& $\left\{e_{1}, e_{2}\right\},\left\{e_{1}, e_{4}\right\}$, \\
& $\left\{e_{2}, e_{4}\right\},\left\{e_{2}, e_{5}\right\}$, \\
\hline 3 & $\left\{e_{1}, e_{2}, e_{3}, e_{4}, e_{5}\right\}$ \\
\hline
\end{tabular}

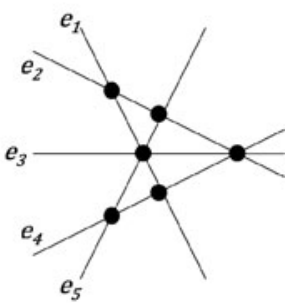

Fig. 1 Point configuration and hyperplane arrangement with corresponding flats

$M$ over $\mathbb{R}$, if and only if there exists an arrangement of hyperplanes whose normal vectors are the $|E|$ columns of $A$. Thus $M$ is representable if and only if $M$ is realized by a hyperplane arrangement. Similarly if a matroid is realized by an affine point set of dimension $d$, then there is an isomorphic vector matroid of rank $d+1$, and $M$ is $\mathbb{R}$-representable. We will thus use the terms representable matroid and realizable matroid interchangeably to mean $\mathbb{R}$-representable.

In Sect. 2.2 we study certain ordinary flats, i.e. rank $r$ flats $F$ that are singleelement extensions of some rank $r-1$ flat $G$. Since the underlying questions there are about the existence of certain point configurations, we need to consider orientability of any discovered matroids, and realizability of any discovered oriented matroids. In order to understand the orientable, but not necessarily realizable case, it will be convenient here to consider the model of oriented matroids provided by arrangements of oriented pseudospheres or oriented pseudohyperplanes. We follow the definitions of Richter-Gebert and Ziegler [23, Chap. 6]. A pseudosphere is the image of a $(d-2)$-sphere $C \subset \mathbb{S}^{d-1}$ under some self homeomorphism of $\mathbb{S}^{d-1}$. An arrangement of pseudospheres is a set of pseudospheres where every subset of $j<d+3$ decompose $\mathbb{S}^{d-1}$ in a way isomorphic to that induced by $j$ linear hyperplanes. We define a projective pseudohyperplane arrangement by identifying antipodal points of a (centrally symmetric representation of a) pseudosphere arrangement. We call vertices of a pseudosphere arrangement simple crossings if all but one of the defining pseudohyperplanes define a 1-face (i.e. a $(d-1)$-flat). We can obtain an oriented matroid from a pseudosphere arrangement as follows. Each pseudosphere partitions $\mathbb{S}^{d-1}$ into two (open) parts; we label one side as + , and one side as - . Given pseudospheres $e_{1} \ldots e_{k}$ forming an arrangement $\mathcal{A}$, each face of $\mathcal{A}$ is thus labeled by some vector in $\{+,-, 0\}^{k}$ (see Fig. 2). These vectors, along with the zero vector, define the covectors of an oriented matroid (see e.g. [4, Chap. 3] for the transformation between the chirotope and the covectors). The topological representation theorem of Folkman and Lawrence [22], shows that every loop-free oriented matroid arises in this way. An oriented matroid $\mathcal{M}$ is called realizable if $\mathcal{M}$ is isomorphic to the oriented matroid of some hyperplane arrangement. As in the matroid case, matrices and affine point sets can be considered as realizing oriented matroids by interpreting them as hyperplane arrangements.

Since orientable matroids thus correspond exactly to classes of M-equivalent pseudohyperplane arrangements, in addition to the specific results discussed here, our enumeration of orientable matroids can be used to confirm arbitrary incidence properties of small pseudohyperplane arrangements. 
Fig. 2 Sign vectors in a (pseudo)hyperplane arrangement

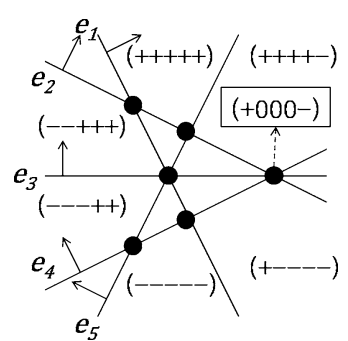

\subsection{On the Points-Lines-Planes Conjecture}

In this section we study the following conjecture of Welsh and Seymour:

Conjecture 1 (Points-lines-planes [38, 40])

(Point configuration version) If $W_{1}$ points in $\mathbb{R}^{3}$ determine $W_{2}$ lines and $W_{3}$ planes, then $W_{2}^{2} \geq(3 / 2)\left(\left(W_{1}-1\right) /\left(W_{1}-2\right)\right) W_{1} W_{3}$ with equality if and only if the points are in general position.

(Matroid version) If $W_{1}, W_{2}$ and $W_{3}$ are the numbers of points, lines and planes of a matroid then $W_{2}^{2} \geq(3 / 2)\left(\left(W_{1}-1\right) /\left(W_{1}-2\right)\right) W_{1} W_{3}$, with equality if and only if every plane contains exactly three points.

Note that the point configuration version is equivalent to a special case of the matroid version where the matroid is rank 4 and realizable.

Originally, Welsh [40] introduced the phrase points-lines-planes conjecture as the point configuration version with a weaker inequality $W_{2}^{2} \geq(3 / 2) W_{1} W_{3}$. Seymour [38] generalized the conjecture to matroids and proved the following special case (an $i^{+}$-point line denotes a line containing $i$ or more points):

Theorem 2 (Seymour [38]) The matroid version of the points-lines-planes conjecture is true for all matroids without a $5^{+}$-point line.

We obtain the following result by enumeration of matroids which do not satisfy the condition in Theorem 2.

Theorem 3 The matroid version of the points-lines-planes conjecture is true for all matroids of rank 4 on $n \leq 11$ elements. Thus, the point configuration version is also true for $n \leq 11$ points.

From Theorem 2 by Seymour, we have only to confirm the inequality for matroids with a $5^{+}$-point line. Although we enumerate matroids of rank 4 only on $n \leq 10$ elements, we can go up to $n=11$ by enumerating only matroids with a $5^{+}$-point line. Indeed, there are only 1,637,504 rank 4 matroids on eleven elements with a $5^{+}$-point line.

This enumeration is obtained as follows. First, note that every single-element deletion of a matroid with a $5^{+}$-point line is a matroid with a $4^{+}$-point line. It follows that enumeration of rank 4 matroids on eleven elements with a $5^{+}$-point line is obtained 
by applying our matroid enumeration algorithm only to rank 4 matroids on ten elements with a $4^{+}$-point line.

\subsection{On the Number of Ordinary Lines and Planes}

The Sylvester-Gallai theorem is known as an outstanding theorem in discrete geometry:

Theorem 4 (Sylvester-Gallai [15, 16, 30, 39])

(Original version) Any configuration of $n \geq 2$ points in a plane, not all of which are on the same line, determines at least one ordinary line that passes through exactly two of the given points.

(Dual version) Any arrangement of $n \geq 2$ lines in a plane, not all of which through the same point, have at least one simple crossing (i.e. crossing of exactly two lines).

Again this theorem can be described in terms of matroids. However, the theorem does not hold for all matroids; the Fano matroid is a counterexample. On the other hand, the theorem also holds for orientable matroids, i.e., pseudoline arrangements in $\mathbb{P}^{2}$.

Let $m_{2}(n)$ and $\tilde{m}_{2}(n)$ be the minimum number of simple crossings in an arrangement of $n$ lines and $n$ pseudolines. The current best lower bound is proven by Csima and Sawyer [10]; $6 n / 13(n \neq 7)$ both for line arrangements and pseudoline arrangements.

For $n$ even $(n \geq 6), n / 2$ is the best possible lower bound for $m_{2}(n)$, because arrangements of $n$ lines with exactly $n / 2$ simple crossings are constructed by Böröczky (see [9]). The arrangements consist of $n / 2$ lines containing the edges of a regular $n / 2$-gon and together with $n / 2$ lines of reflective symmetry. Böröczky's example for $n=12$ is shown in Fig. 3. Moreover, for $n=12$, another arrangement of $n$ lines with $n / 2$ simple crossings is known as shown in Fig. 4 (together with the line at infinity, Fig. 4 is the "McKee configuration" of 13 lines with six simple crossings) [9].

Hansen [27] conjectured that the arrangement shown in Fig. 4 and one by Böröczky are the only line arrangements with $n / 2$ simple crossings. We proved Hansen's conjecture for $n \leq 12$; in fact, we obtained the following stronger result on pseudoline arrangements:

Theorem 5 For $n \leq 12$ even, there are no arrangements of $n$ lines with exactly $n / 2$ simple crossings other than the arrangement of 12 lines shown in Fig. 4 and Böröczky's arrangements. Furthermore, the same statement is also true for arrangements of pseudolines.

We describe how we obtain this result. Recall that simple crossings in pseudoline arrangements correspond to 2-point lines in orientable matroids. First, we enumerate all orientable matroids on $n$ elements with $n / 22$-point lines. As a result, we determine that there is one such matroid for $n=6,8,10$ and two for $n=12$. Next, we generate all orientations of them and obtain the result that each of these matroids has 
Fig. 3 Böröczky's arrangement of $n=12$ lines

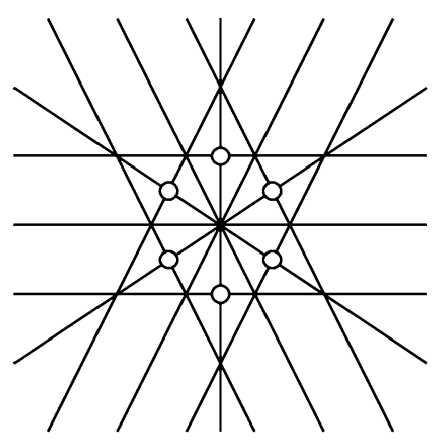

Fig. 4 Another arrangement of twelve lines with six simple crossings (two of them are at infinity line)

\begin{tabular}{llllllll}
\hline$n$ & 4 & 5 & 6 & 7 & 8 & 9 & 10 \\
\hline$m_{3}(n)$ & 4 & 5 & 6 & 7 & 8 & 6 & 8 \\
$\tilde{m}_{3}(n)$ & 4 & 5 & 6 & 7 & 8 & 6 & 8 \\
\hline
\end{tabular}

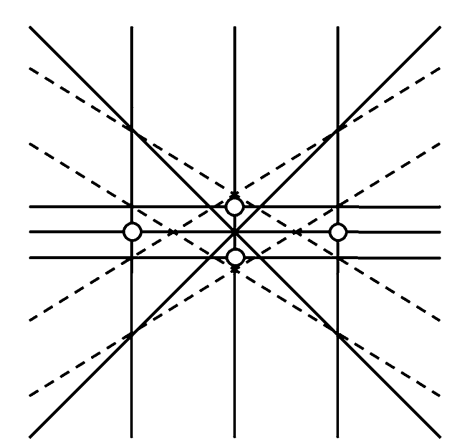

0
Table 3 Minimum number of ordinary planes in configurations of $n$ points in $\mathbb{R}^{3}$ $\left(m_{3}(n)\right)$ and rank 4 oriented matroids on $n$ elements $\left(\tilde{m}_{3}(n)\right)$

unique orientation up to isomorphism. Finally, we confirm that they correspond to line arrangements, as stated in Theorem 5.

In three or more dimensions, there may not be a hyperplane containing the minimum possible number of points; consider two skew lines each with three points. Motzkin [32] defined an ordinary hyperplane in $d$ dimensions as one with all but one contained points in a $(d-2)$-flat, and showed that any non-collinear point configuration in $\mathbb{P}^{3}$ contains an ordinary plane (Hansen [26] generalized this to $\mathbb{P}^{d}$ ). In (oriented) matroid terms, define an ordinary hyperplane as a $d$-flat that is a singleelement extension of a $(d-1)$-flat. Let $m_{3}(n)$ and $\tilde{m}_{3}(n)$ be the minimum number of ordinary planes in a configuration of $n$ points in $\mathbb{P}^{3}$ and in a rank 4 oriented matroid on $n$-elements, respectively. We obtain $m_{3}(n)$ and $\tilde{m}_{3}(n)$ for $n \leq 10$ and summarize the result in Table 3 . Note that we do not need to determine orientability of all rank 4 matroids on ten elements because we have only to investigate those with at most 10 ordinary planes.

For $n \leq 8, m_{3}(n)=\tilde{m}_{3}(n)=n$, but not for $n=9$ and 10. In the same way as the 2-dimensional case, we determine cases giving $m_{3}(n)$ and $\tilde{m}_{3}(n)$ for $n=9$ and 10. In both cases, a unique $\mathbb{R}$-representable oriented matroid, i.e., a point configuration in 
Fig. 5 Configuration nine points with six ordinary planes: $A B I, C D I, A F G, B E H$, $C G H, D E F$

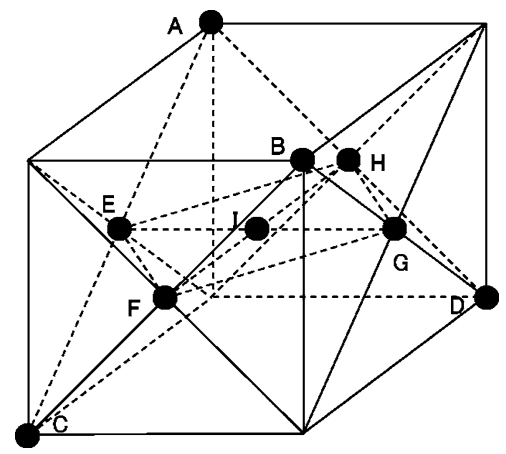

Fig. 6 Configuration of ten points with eight ordinary planes: $A C F, A C H, B D E$, $B D G, A F H, C F H, B E G$, $D E G$

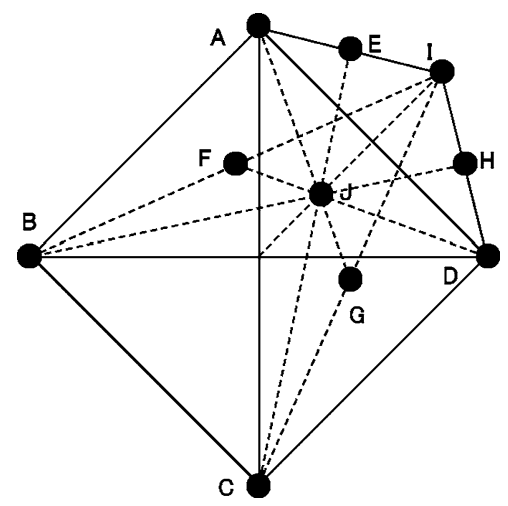

$\mathbb{R}^{3}$ gives the lower bound. We show these extremal examples in Figs. 5 and 6 . These are the first examples satisfying $m_{3}(n)=\tilde{m}_{3}(n)<n$.

\section{Matroid Enumeration}

In this section, we describe our matroid enumeration algorithm and present the computational result. Section 3.1 introduces the basic notions and terminology. Section 3.2 explains the characterization of all possible single-element extensions in terms of so-called modular cuts, which encode the incidence of the new element. Section 3.3 presents the outline of the enumeration algorithm. Sections 3.4 and 3.5 describe two key elements of the algorithm. Section 3.6 presents the results of matroid enumeration.

Firstly, we describe the idea of our matroid enumeration algorithm and its features in comparison with other algorithms. Blackburn, Crapo and Higgs's algorithm [5] gives a basis for Mayhew and Royle's [28] and ours. The key in their algorithm is construction of matroids on $n$ elements from one on $n-1$ elements by a singleelement extension, which is the reverse of deletion of an element from a matroid. Starting from the root matroid on the empty set, all matroids are generated by repeated single-element extensions and elimination of extra isomorphic copies. We show a step in their algorithm in the left of Fig. 7. 

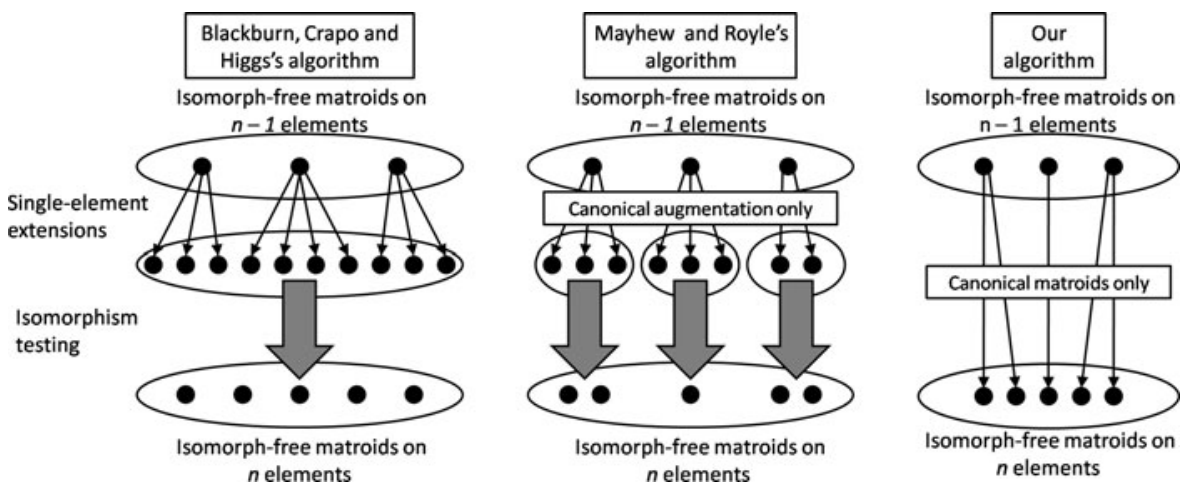

Fig. 7 Difference between the three matroid enumeration algorithms from an isomorphism testing standpoint

When the number of generated matroids becomes large, isomorphism testing becomes a bottleneck. In general, for efficient isomorph-free enumeration of combinatorial objects, a desirable algorithm is one which produces one object in each isomorphism class without pairwise isomorphism testing. Such algorithms are called orderly algorithms (Reed [34] introduced this term first in a slightly more restricted sense). Orderly algorithms are memory efficient and easy to parallelize. However, constructing orderly algorithms for individual problems is not necessarily easy.

There are several approaches to construct orderly algorithms. One is the canonical representation orderly algorithm, developed by Read [34] and Faradzev [17] independently and also called Read/Faradzev-type orderly algorithm. Our matroid enumeration algorithm falls into this class of algorithm. For enumeration of oriented matroids (a signed extension of matroids) Finschi and Fukuda [18-20] succeeded in this approach. This encourages us to apply their idea to enumeration of matroids. Figure 8 shows the idea of our algorithm. In the canonical representation orderly algorithm, a canonically labeled object called the canonical object is chosen as the representation of each isomorphism class. A key in the canonical representation algorithm is the choice of canonical objects. It must satisfy the following condition: a canonical object must be generated from another canonical object via an augmentation, which is a single-element extension in a case of matroid enumeration. This condition ensures all canonical objects are generated. We propose as a choice of canonical matroids an unsigned version of the canonical oriented matroids used by Finschi and Fukuda [18, 19] which in-turn are an encoding of oriented matroids by its chirotope with reverse lexicographic order of $r$-subsets of the ground set.

There are other approaches to orderly algorithms. One is the canonical construction path orderly algorithm, developed by McKay [29] and also called the McKaytype orderly algorithm. Mayhew and Royle's matroid enumeration algorithm [28] is based on this approach. In the canonical construction path algorithm, only objects generated via a canonical augmentation are output. The canonical construction path orderly algorithm shares the idea of traversing the tree with the canonical representation orderly algorithm but differs in that it does not define canonically labeled objects in each isomorphism class. Unlike the canonical representation algorithm, it is essen- 
Fig. 8 Canonical representation orderly algorithm

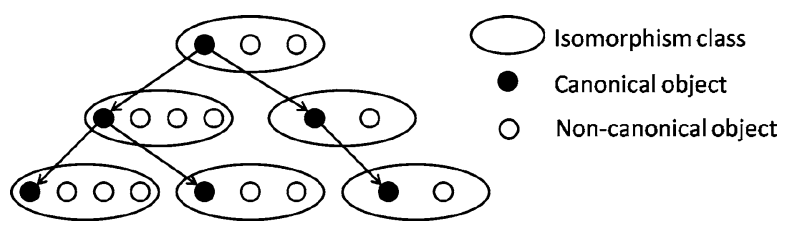

tially unlabeled and intermediate objects may be relabeled freely. On the other hand, if an object has multiple isomorphic augmentations, they cannot be distinguished by the technique of canonical augmentation. Extra isomorphic augmentations must be rejected in another way. In a case of matroids, direct rejection of isomorphic singleelement extensions is difficult because of their complex structure. Therefore, Mayhew and Royle took a two-step approach as shown in the middle of Fig. 7. They allow isomorphic single-element extensions at a step of generating single-element extensions and eliminate extra ones by pairwise isomorphism testing. Because of this isomorphism testing, they call their algorithm partially orderly. Although this isomorphism testing is restricted to matroids that are generated as single-element extensions of the same matroid and accepted as canonical augmentations, it still becomes a bottleneck when the number of such matroids is large. In comparison, our algorithm does not need such pairwise isomorphism testing at all as illustrated in the right of Fig. 7. Thus our algorithm can be called completely orderly. A canonical labeling orderly algorithm is usually superior for cases where it is difficult to reject multiple isomorphic augmentations.

Our algorithm has several specific improvements to the generic canonical representation orderly algorithm. By exploiting the properties of our canonical labeling, we prune the search of non-canonical single-element extensions. This pruning is based on the fact that if a single-element extension corresponds to a modular cut (a special collection of flats, see Sect. 3.2) containing a certain type of flats, it is non-canonical. We call these flats taboo flats. This pruning suggests a unified approach for improvement of the canonical representation algorithms using the properties of canonical labellings.

\subsection{Matroid Background}

In this section we supplement the discussion of matroids in Sect. 2 with some further definitions and notation that will be useful below.

As we mentioned in Sect. 2, we regard a matroid as an ordered pair $(E, r)$ where $E$ is a ground set and $r: 2^{E} \rightarrow \mathbb{Z}$ is a rank function. We add the following definitions to those in Sect. 2. $X \subseteq E$ is independent if $|X|=r(X)$, otherwise dependent. Similarly, if a flat $X$ such that $\operatorname{cl}(X)=X$ is dependent, it is called a dependent flat. A set $X \subseteq E$ is a basis if $|X|=r(X)=r(M)$. Given a non-negative integer $m \leq|E|$, if every subset $X \subseteq E$ such that $|X| \leq m$ is independent (and those are the only independent sets), $M$ is called the uniform matroid of rank $m$. A minimal dependent set $X \subseteq E$ is a circuit. If $M$ has no circuits $X \subseteq E$ such that $|X|<r(X), M$ is called a paving matroid. $e \in E$ is a loop if $r(e)=0$ and $e, f \in E$ are parallel if $r(e \cup f)=1$. A matroid is simple if it contains no loops or parallel pairs of elements. $e \in E$ is a coloop if every base contains $e$. 
For $T \subseteq E$, define a function $r^{\prime}(X)$ for all $X \subseteq E-T$ as $r^{\prime}(X):=r(X \cup T)-$ $r(T) . M^{\prime}=\left(E-T, r^{\prime}\right)$ is a matroid, called the contraction of $M$ to $E-T$. Given a function $r^{\prime \prime}(X):=r(X)$ for all $X \subseteq E-T, M^{\prime \prime}=\left(E-T, r^{\prime \prime}\right)$ is a matroid, called the deletion of $T$ from $M$. For the deletion of $e \in E$ from $M$, denoted by $M \backslash e$, we say that $M$ is a single-element extension of $M \backslash e$. Given two matroids $M$ and $N$, $N$ is called a minor of $M$ if it is obtained from $M$ by a sequence of restriction and contraction operations.

\subsection{Modular Cuts and Single-Element Extensions}

Crapo [8] developed the notion of single-element extensions and found that they are in one-to-one correspondence with special subsets of flats called modular cuts. In the point configuration setting, a modular cut is just the set of flats containing the newly inserted point (cf. Fig. 10). To motivate the axiomatic definition in terms of rank functions, we make the following observation:

Lemma 1 If $F_{1}$ and $F_{2}$ are flats of matroid $M$, and

1. $r\left(F_{1} \cup\{e\}\right)=r\left(F_{1}\right), r\left(F_{2} \cup\{e\}\right)=r\left(F_{2}\right)$, and

2. $r\left(F_{1}\right)+r\left(F_{2}\right)=r\left(F_{1} \cup F_{2}\right)+r\left(F_{1} \cap F_{2}\right)$

then $r\left(\left(F_{1} \cap F_{2}\right) \cup\{e\}\right)=r\left(F_{1} \cap F_{2}\right)$

Proof Let $\hat{F}_{i}=F_{i} \cup\{e\}$. From the submodularity property of rank functions, we have

$$
\begin{aligned}
r\left(\left(F_{1} \cap F_{2}\right) \cup\{e\}\right) & \leq r\left(\hat{F}_{1}\right)+r\left(\hat{F}_{2}\right)-r\left(\hat{F}_{1} \cup \hat{F}_{2}\right) \\
& \leq r\left(F_{1}\right)+r\left(F_{2}\right)-r\left(F_{1} \cup F_{2}\right) \\
& =r\left(F_{1} \cap F_{2}\right)
\end{aligned}
$$

where the last inequality follows from the second condition of the lemma.

Pairs of flats satisfying the second condition of Lemma 1 are called modular (as opposed to strictly submodular). In point configuration terms (see Fig. 9), Lemma 1 says that if $(A, B)$ are a modular pair, and $e \in \operatorname{aff}(A) \cap \operatorname{aff}(B)$ then $e \in \operatorname{aff}(A \cap B)$ (where aff is the affine hull).

Definition 1 (Crapo [8], Oxley [33]) Let $\mathcal{F}(M)$ be the set of flats of a matroid $M$. $\mathcal{A} \subseteq \mathcal{F}(M)$ is called a modular $c u t$ if $\mathcal{A}$ satisfies the following two conditions:

(i) If $F \in \mathcal{A}$ and $F^{\prime}$ is a flat of $M$ containing $F$, then $F^{\prime} \in \mathcal{A}$.

(ii) If $F_{1}, F_{2} \in \mathcal{A}$ and $r\left(F_{1} \cup F_{2}\right)+r\left(F_{1} \cap F_{2}\right)=r\left(F_{1}\right)+r\left(F_{2}\right)$, then $F_{1} \cap F_{2} \in \mathcal{A}$.

The following Lemma shows that every single-element extension yields a modular cut, i.e. that the set of flats containing the extension element satisfies Definition 1. 
Fig. 9 Pairs of 2-flats in a rank 4 matroid. The right pair is modular, i.e.

$r\left(F_{1} \cup F_{2}\right)+r\left(F_{1} \cap F_{2}\right)=$ $r\left(F_{1}\right)+r\left(F_{2}\right)$
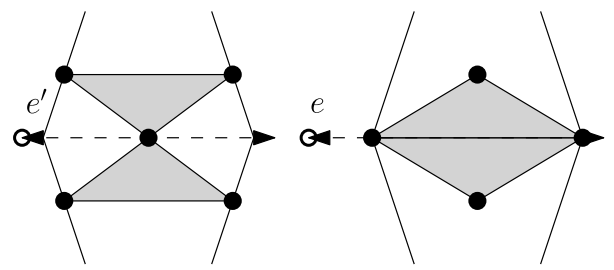

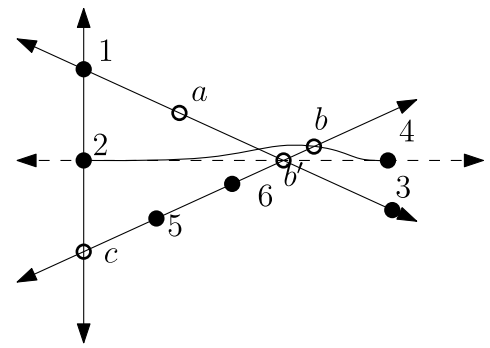

$\{1,3\},\{1 \ldots 6\}$

$\{1,3\},\{5,6\},\{1 \ldots 6\}$

$\{1,3\},\{2,4\},\{5,6\},\{1 \ldots 6\}$

$\{1,2\},\{5,6\},\{1 \ldots 6\}$

Fig. 10 Illustrating the correspondence between modular cuts and single-element extensions

Lemma 2 (Crapo [8], Oxley [33]) Let $N$ be a single-element extension of a matroid $M$, and $\mathcal{F}(M)$ and $\mathcal{F}(N)$ the set of flats of a matroid $M$ and $N$, respectively. If $F \in \mathcal{F}(M)$ satisfies the following condition, $M$ is a modular cut.

$$
F \cup e \in \mathcal{F}(N) \text { and } \quad \mathrm{r}(\mathrm{F} \cup \mathrm{e})=\mathrm{r}(\mathrm{F})
$$

Figure 10 illustrates the relation between modular cuts and single-element extensions. Here we have six points $\{1, \ldots, 6\}$, and make single-element extensions by $a$, $b, b^{\prime}$, and $c$. We list modular cuts corresponding to each single-element extension.

The following Theorem, along with Lemma 2, gives a one-to-one correspondence between single-element extensions and modular cuts. Thus we can generate all single-element extensions of a matroid $M$ by enumerating all modular cuts of $M$.

Theorem 6 (Crapo [8], Oxley [33]) Let $\mathcal{A}$ be a modular cut of a matroid $M$ on a set $E$. Then there is a unique extension Nof $M$ on $E \cup$ e such that $\mathcal{A}$ consists of those flats $F$ of $M$ for which $F \cup e$ is a flat of $N$ having the same rank as $F$. The following conditions are satisfied for all subsets $X$ of $E$ :

$$
r_{N}(X \cup e)= \begin{cases}r_{M}(X), & \text { if } c l_{M}(X) \in \mathcal{A}, \\ r_{M}(X)+1, & \text { if } c l_{M}(X) \notin \mathcal{A} .\end{cases}
$$

$r_{M}$ and $r_{N}$ are rank functions of matroids $M$ and $N$, respectively.

We denote the matroid $N$ in the above theorem by $M+{ }_{\mathcal{A}} e$. Note that $r\left(M+{ }_{\mathcal{A}} e\right)=$ $r(M)+1$ if and only if $\mathcal{A}=\emptyset$ and $r(M+\mathcal{A} e)=r(M)$ otherwise. 


\subsection{Outline of Pruned Canonical Representation Orderly Algorithm for Matroid Enumeration}

In this section, we describe the outline of our pruned canonical representation orderly algorithm for matroid enumeration.

In the following, we concentrate on generating a list of (canonical) isomorph-free matroids in $\operatorname{IC}(n, r)$ under the assumption that canonical representatives for $\operatorname{IC}(n-$ $1, r-1)$ and $\operatorname{IC}(n-1, r)$ are known. This is because we can complete the entire enumeration by iterating this step with increasing $n$ and $r$.

To implement a canonical representation orderly algorithm, we need to choose a canonical matroid in each isomorphism class. satisfying the following condition: each canonical matroid is generated as a single-element extension of a (smaller) canonical matroid. We describe the details of the choice of canonical matroids in Sect. 3.4.

The remaining problem is generating all single-element extensions of a matroid. This can be done with a simple backtracking search on the set of flats. However this generates many non-canonical matroids which we discard. We propose pruning the search by exploiting properties of our choice of canonical matroids. In Sect. 3.5 we identify several families of taboo flats $F$ where any modular cut $\mathcal{A}$ containing $F$ determines a non-canonical single-element extension. By definition, we have only to search for modular cuts without taboo flats. We describe some types of taboo flat which can be determined directly in Sect. 3.5.

In the following, we suppose that the ground set of a matroid on $n$ elements is $E_{n}=\left\{e_{1}, \ldots, e_{n}\right\}$. We present the outline of the pruned canonical representation algorithm in Algorithm 1.

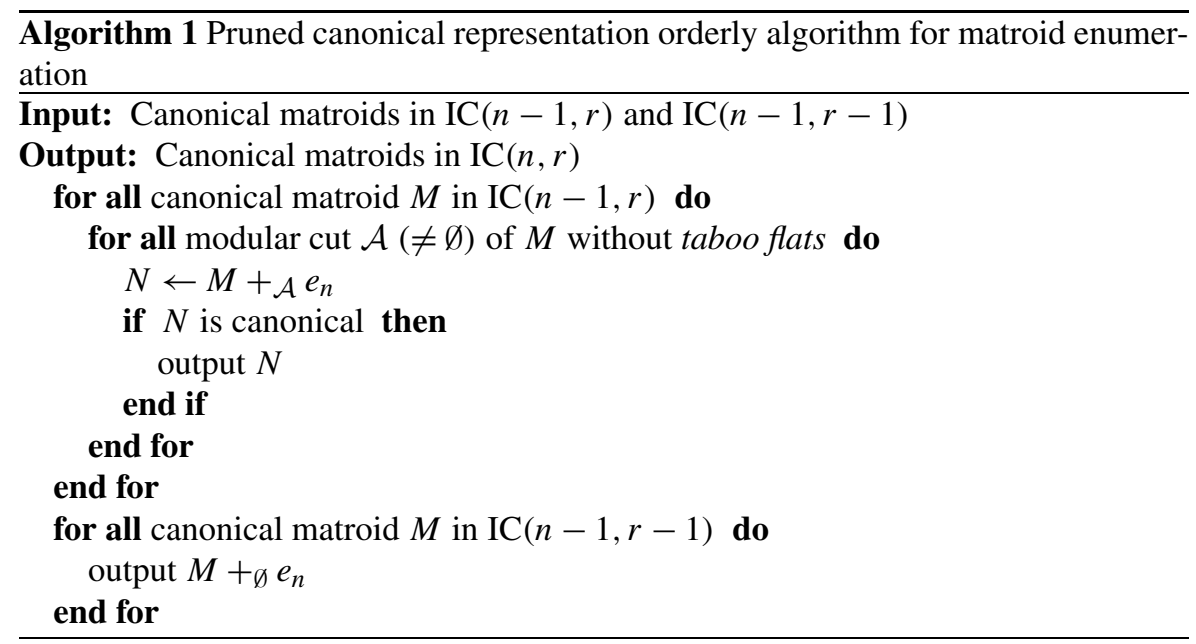

Details of the choice of canonical matroids and finding taboo flats are discussed in Sects. 3.4 and 3.5, respectively. Our current implementation uses straightforward orbit traversal to test matroids for canonicality. This method is fast enough for the cases we tested. 
Parallelizing enumeration algorithms has been extensively studied, and sophisticated approaches have been developed (see e.g. [7]). Here, we can take a simpler approach. We can easily parallelize our algorithm by dividing a task into computing canonical extensions of each matroid in $\operatorname{IC}(n-1, r-1)$ and $\operatorname{IC}(n-1, r)$. Because the cost of each divided task is balanced to some extent in our experiments, this direct parallelization works well.

\subsection{Canonical Representation of Matroids}

In this section, we describe how we choose a canonical matroid for each isomorphism class of matroids. In particular we introduce an encoding of matroids based on the encoding of oriented matroids developed by Finschi and Fukuda [18, 19]. A matroid $M$ is determined by the set of bases (i.e. minimal sets of rank $r$ ). Let $M$ be a rank $r$ matroid on $E_{n}=\left\{e_{1}, e_{2}, \ldots, e_{n}\right\}$. Because all bases of $M$ have the same cardinality $r$, we can represent the set of bases by describing whether each $r$-subset of $E_{n}$ is a basis or not. Consequently, a matroid is encoded as a list of $\left(\begin{array}{l}n \\ r\end{array}\right)$ Boolean values corresponding to the $r$-subsets of $E_{n}$ in some canonical order. We choose the reverse lexicographic order of the $r$-subsets as follows.

Definition 2 (Reverse Lexicographic Order of Sets) The reverse lexicographic order of sets is defined as follows: for given distinct $r$-sets $A$ and $B, A \prec B$ if $\max (A)<$ $\max (B)$ or $\max (A)=\max (B)=e_{m}$ and $A \backslash\left\{e_{m}\right\} \prec B \backslash\left\{e_{m}\right\}$.

Definition 3 (Encoding) Suppose that an asterisk (' $*$ ') means the corresponding $r$ subset is a basis and a zero (' 0 ') means otherwise. The encoding of a matroid $M$ of rank $r$ on $E_{n}$ is a list composed of $\left(\begin{array}{l}n \\ r\end{array}\right)$ symbols ' $*$ ' or a zero ' 0 ' where symbol $j$ corresponds to the $j$ th $r$-subset in reverse lexicographic order.

Figure 11 shows a matroid with the geometric representation and its encoding “* $0 * * * 0 * * * *$ ". We choose a canonical matroid in each isomorphism class based on the preceding two definitions.

Definition 4 (Canonical Matroid) A canonical matroid of the isomorphism class is one with the lexicographically smallest encoding within the isomorphism class where symbols are ordered $*>0$. For a given matroid $M, \operatorname{rep}(M)$ denotes the canonical matroid the isomorphic to $M$.

The canonical matroid which is isomorphic to the matroid in Fig. 11 is the one encoded as " $0 * * * 0 * * * * *$ ", which is obtained by an isomorphism exchanging $e_{3}$ and $e_{4}$. The next theorem ensures that our choice of canonical matroids satisfies the condition that a canonical matroid is generated as another single-element extension of a canonical matroid.

Theorem 7 Let $M$ be a canonical matroid of rank $r$ on $E_{n}$.

1. If $e_{n}$ is not a coloop in $M, M \backslash e_{n}$ is a matroid of rank $r$ on $n-1$ elements which is encoded as the first $\left(\begin{array}{c}n-1 \\ r\end{array}\right)$ symbols of the encoding of $M$. Furthermore, $M \backslash e_{n}$ is canonical. 
Fig. 11 A matroid, its bases in the reverse lexicographic order of $r$-subsets and its encoding

\begin{tabular}{|l|l|}
\hline$\left\{e_{1}, e_{2}, e_{3}\right\}$ & base \\
\hline$\left\{e_{1}, e_{2}, e_{4}\right\}$ & non-base \\
\hline$\left\{e_{1}, e_{3}, e_{4}\right\}$ & base \\
\hline$\left\{e_{2}, e_{3}, e_{4}\right\}$ & base \\
\hline$\left\{e_{1}, e_{2}, e_{5}\right\}$ & base \\
\hline$\left\{e_{1}, e_{3}, e_{5}\right\}$ & non-base \\
\hline$\left\{e_{2}, e_{3}, e_{5}\right\}$ & base \\
\hline$\left\{e_{1}, e_{4}, e_{5}\right\}$ & base \\
\hline$\left\{e_{2}, e_{4}, e_{5}\right\}$ & base \\
\hline$\left\{e_{3}, e_{4}, e_{5}\right\}$ & base \\
\hline
\end{tabular}

1112112123 2233233444 3444555555 $* 0 * * * 0 * * * *$

2. If $e_{n}$ is a coloop in $M, M \backslash e_{n}$ is a matroid of rank $(r-1)$ on $n-1$ elements which is encoded as the last $\left(\begin{array}{l}n-1 \\ r-1\end{array}\right)$ symbols of the encoding of $M$. Furthermore, $M \backslash e_{n}$ is canonical.

Proof First, we prove the rank and encoding in each case.

If $e_{n}$ is not a coloop, there are bases of $M$ which do not contain $e_{n}$. Because they are also bases of $M \backslash e_{n}$, the rank of $M \backslash e_{n}$ is $r$. By definition of the ordering of bases, the encoding of $M \backslash e_{n}$ is the first $\left(\begin{array}{c}n-1 \\ r\end{array}\right)$ symbols of the encoding of $M$.

If $e_{n}$ is a coloop, then the rank of $M \backslash e_{n}$ is $r-1$ because all bases of $M$ contain $e_{n}$. In particular the size of the maximal independent set is $r-1$, so the rank of $M \backslash e_{n}$ is $r-1$. By definition of the ordering of bases, the encoding of $M \backslash e_{n}$ is the last $\left(\begin{array}{l}n-1 \\ r-1\end{array}\right)$ symbols of the encoding of $M$.

Next, we prove the second part of the theorem. Suppose that $M \backslash e_{n}$ is noncanonical. In this case, there exists an isomorphism on $E_{n} \backslash e_{n}$ which makes the encoding of $M \backslash e_{n}$ larger. This isomorphism also makes the encoding of $M$ larger. This contradicts the supposition that $M$ is canonical.

\subsection{Taboo Flats for Pruning Search of Single-Element Extensions}

In this section, we describe several types of taboo flat for implementing the pruned generation of single-element extensions. We give the following formal definition of taboo flats:

Definition 5 (Taboo Flat) A flat $F$ of a matroid $M$ is a taboo flat if the following condition is satisfied: for all modular cuts $\mathcal{A}$ containing $F$, a single-element extension determined by $\mathcal{A}$ is non-canonical.

These types of taboo flat arise from the properties of our definition of canonical matroids. The first type of taboo flat is given by the following proposition.

Proposition 1 Let $M$ be a canonical rank $r$ matroid on $E_{n-1}$, and $\mathcal{A}$ a modular cut of $M$. If $F \in \mathcal{A}$ is a rank $r-1$ dependent flat of $M$ of maximum cardinality, then $F$ is a taboo flat.

Proof Let $z(M)$ be the number of leading zeros in the encoding of $M$. If $G$ is a rank $(r-1)$ flat of $M$ and $|G| \geq r, z(M) \geq\left(\begin{array}{c}|G| \\ r\end{array}\right)$ because $M$ is canonical (consider the 
isomorphism that maps $G$ to $\left.\left\{e_{1}, \ldots, e_{|G|}\right\}\right)$. Because $F$ is a maximum cardinality rank $(r-1)$ flat and $|F| \geq r,\left(\begin{array}{c}|F| \\ r\end{array}\right) \leq z(M)<\left(\begin{array}{c}|F|+1 \\ r\end{array}\right)$. From Theorem 6, the rank of $F \cup e_{n}$ is $r-1$ in $N$. Similarly, $\left(\begin{array}{c}|F+1| \\ r\end{array}\right) \leq z(\operatorname{rep}(N))<\left(\begin{array}{c}|F+2| \\ r\end{array}\right)$. Because by Theorem 7 $z(M)=z(N)$, it follow that $z(N) \neq z(\operatorname{rep}(N))$, thus $N$ is non-canonical.

Next, we describe the second type of taboo flats.

Proposition 2 Let $M$ be a canonical matroid on $E_{n-1}$, and $\mathcal{A}$ a modular cut of $M$. We denote a family of $r$-subsets of $E_{n-1}$ which contains $e_{n-1}$ by $R$, and let $\mathcal{D}$ be the subset of $R$ before the first dependent flat under the reverse lexicographic order. If $F \in \mathcal{A}$ is a flat with $E \cup\left\{e_{n-1}\right\} \in \mathcal{D}$ for some $E \subset F$ such that $r(E)=R(F)$, then $F$ is a taboo flat.

Proof Consider the isomorphism that exchanges $e_{n-1}$ and $e_{n}$. This isomorphism does not change the first $\left(\begin{array}{c}n-2 \\ r\end{array}\right)$ symbols in the encoding of $N$, so we focus on the remaining $\left(\begin{array}{l}n-2 \\ r-2\end{array}\right)+2\left(\begin{array}{l}n-2 \\ r-1\end{array}\right)=\left(\begin{array}{l}n-2 \\ r-1\end{array}\right)+\left(\begin{array}{l}n-1 \\ r-1\end{array}\right)$ symbols. Since by Theorem $6, E \cup\left\{e_{n}\right\}$ is dependent, the isomorphism moves the location of the first zero in these symbols into the front. Consequently, $N$ is non-canonical.

Finally, we remark the following.

Proposition 3 A sub-flat of a taboo flat is also a taboo flat.

Proof Suppose that $F$ is a taboo flat and $G \subseteq F$ is a flat. From Definition $1, G \in \mathcal{M}$ implies $F \in \mathcal{M}$.

The three preceding propositions imply that the ground set $E_{n-1}$ cannot be a taboo flat while the empty set \{\} is always a taboo flat.

As an example, we consider taboo flats of the matroid in Fig. 12. Because $\left\{e_{1}, e_{2}, e_{3}, e_{4}\right\}$ is the largest dependent flat of rank 2 , it is a taboo flat according to Proposition 1. From Proposition 2, flats which contain $\left\{e_{1}, e_{2}\right\},\left\{e_{1}, e_{3}\right\},\left\{e_{2}, e_{3}\right\}$, $\left\{e_{1}, e_{4}\right\},\left\{e_{2}, e_{4}\right\},\left\{e_{3}, e_{4}\right\}$ or $\left\{e_{1}, e_{5}\right\}$ are taboo flats. This means $\left\{e_{1}, e_{2}, e_{3}, e_{4}\right\}$ and $\left\{e_{1}, e_{5}, e_{6}\right\}$ are taboo flats. From Proposition 3, $\left\{e_{1}\right\},\left\{e_{2}\right\},\left\{e_{3}\right\},\left\{e_{4}\right\},\left\{e_{5}\right\},\left\{e_{6}\right\}$, and \{\} are also taboo flats. All taboo flats are shown in Fig. 12 as broken line boxes.

\subsection{Result of Matroid Enumeration}

We used Algorithm 1 to enumerate isomorph-free rank $r$ matroids on $n$ elements for $r \leq 3, n \leq 12$ and for $r=4, n \leq 10$. For $r \geq n / 2$, the counts are obtained by matroid duality. Table 1 summarizes our results. We used 24 processes on 12 dualcore processors (Opteron $2.1 \mathrm{GHz}$ ) for calculation and it took about 3 days. If we sum up computation time of each processor, it is 79 days. Our parallelization by dividing a task into extensions of each matroid works well because no subtask is extremely difficult. In our experiments, all subtasks finish in less than 10 minutes.

The number of matroids of a give rank and number of elements is summarized in Table 1. The numbers are in complete agreement with previous results. Welsh [40] 


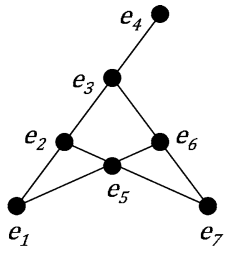

11121121231121231234112123123412345 22332334442334445555233444555566666 34445555556666666666777777777777777 $0000^{* * * * * * * * * * * *} 0 * * * * * * * * * * 0 * * * * 0 * *$

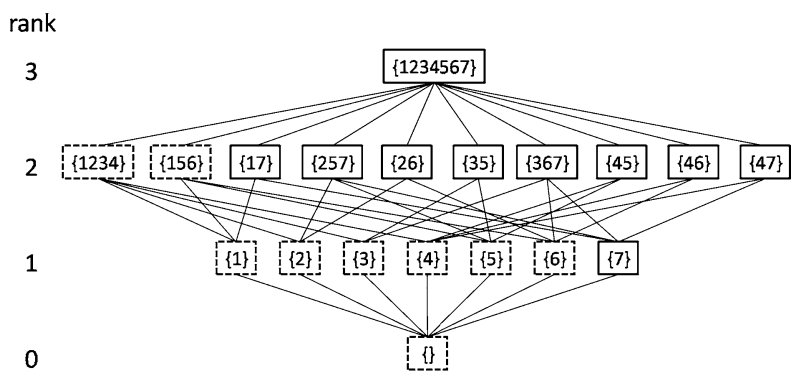

Fig. 12 A matroid and the lattice of its flats (taboo flats are broken line boxes)

Table 4 Number of non-isomorphic simple matroids

\begin{tabular}{|c|c|c|c|c|c|c|c|c|c|c|c|c|c|}
\hline$r \backslash n$ & 0 & 1 & 2 & 3 & 4 & 5 & 6 & 7 & 8 & 9 & 10 & 11 & 12 \\
\hline 0 & 1 & & & & & & & & & & & & \\
\hline 1 & & 1 & & & & & & & & & & & \\
\hline 2 & & & 1 & 1 & 1 & 1 & 1 & 1 & 1 & 1 & 1 & 1 & 1 \\
\hline 3 & & & & 1 & 2 & 4 & 9 & 23 & 68 & 383 & 5249 & 232928 & 28872972 \\
\hline 4 & & & & & 1 & 3 & 11 & 49 & 617 & 185981 & 4884573865 & $*$ & * \\
\hline 5 & & & & & & 1 & 4 & 22 & 217 & 188936 & $*$ & $*$ & * \\
\hline 6 & & & & & & & 1 & 5 & 40 & 1092 & 4886374072 & $*$ & * \\
\hline 7 & & & & & & & & 1 & 6 & 66 & 9742 & $*$ & * \\
\hline 8 & & & & & & & & & 1 & 7 & 104 & 298034 & * \\
\hline 9 & & & & & & & & & & 1 & 8 & 156 & 31898447 \\
\hline 10 & & & & & & & & & & & 1 & 9 & 229 \\
\hline 11 & & & & & & & & & & & & 1 & 10 \\
\hline 12 & & & & & & & & & & & & & 1 \\
\hline
\end{tabular}

Note: $r$ : rank $n$ : size of the ground set

*Non-determined value

asked the question of whether most matroids are paving. Mayhew and Royle showed paving matroids predominate in matroids on nine elements. $71.71 \%$ of matroids on nine elements are paving, compared to $49.50 \%$ of matroids on eight elements. Mayhew and Royle [28] showed the total number of paving matroids of rank 4 on ten elements is $4,528,127,429$. Here we determine all matroids of rank 4 on ten elements including non-paving ones, which shows $92.6 \%$ of rank 4 matroids on ten elements are paving. Compared to $77.36 \%$ of rank 4 matroids on nine elements [36], our result also gives additional evidence that paving matroids do indeed predominate.

Some previous studies on enumeration of matroids focus on simple matroids, including the result of Blackburn, Crapo and Higgs on matroids on $n \leq 8$ elements [5] and one of Betten and Betten on rank 3 matroids on $n \leq 12$ elements [3]. We also show the number of simple matroids in Table 4 and our results are in complete agreement with these previous results. 


\section{Orientations of Matroids}

In this section, we consider the orientability of the matroids enumerated in Sect. 3. Section 4.1 introduces the basic notions on orientations. As mentioned in the introduction, orientability of matroids is known to be NP-complete [35], and all known methods are unsurprisingly exponential time in the worst case. Experimental evaluations are thus crucial. Section 4.2 presents our method for testing orientability and some experimental results. The experimental results are discussed in more detail in Sect. 4.3, which investigates minimal non-orientable matroids and the strength of existing non-orientability certificates.

\subsection{Oriented Matroids}

Oriented matroids have been studied as a signed refinement of matroids where each basis is given a sign in a way consistent with e.g. the signs of determinants of full rank submatrices (we give formal definitions below). There are many equivalent axiomatizations of oriented matroids (see [4, Chap. 0] or [23, Chap. 6] for a survey). In this paper, we find the chirotope axioms the most convenient. A chirotope is a mapping which represents signed bases. A formal definition is as follows.

Definition 6 Let $E$ be a finite set and $r$ be an integer $0 \leq r \leq|E|$. An alternating mapping $\chi: E^{r} \rightarrow\{-1,0,1\}$ is a chirotope if it satisfies the following two conditions.

(i) The set of $r$-subsets $\left\{x_{1}, x_{2}, \ldots, x_{r}\right\}$ of $E$ such that $\chi\left(x_{1}, x_{2}, \ldots, x_{r}\right) \neq 0$ is the set of bases of a matroid of rank $r$ on $E$.

(ii) [3-term Grassman-Plücker relations] For any $x_{3}, \ldots, x_{r}, y_{1}, y_{2}, z_{1}, z_{2} \in E$,

if $\chi\left(z_{1}, y_{2}, x_{3}, \ldots, x_{r}\right) \cdot \chi\left(y_{1}, z_{2}, x_{3}, \ldots, x_{r}\right) \geq 0$

and $\chi\left(z_{2}, y_{2}, \ldots, x_{r}\right) \cdot \chi\left(z_{1}, y_{1}, x_{3}, \ldots, x_{r}\right) \geq 0$

then $\chi\left(y_{1}, y_{2}, x_{3}, \ldots, x_{r}\right) \cdot \chi\left(z_{1}, z_{2}, x_{3}, \ldots, x_{r}\right) \geq 0$.

Each oriented matroid has two opposite chirotopes, $\chi$ and $-\chi$. Formally, we define an oriented matroid as an ordered pair $(E,\{\chi,-\chi\})$ of a ground set $E$ and the set of two opposite chirotopes $\{\chi,-\chi\}$. Negation of a chirotope $\chi$ into $-\chi$ gives an identical oriented matroid. Because a chirotope is alternating, it is determined with $\left(\begin{array}{c}|E| \\ r\end{array}\right)$ values on $r$-subsets of $E$ if some canonical ordering on $E$ is given.

If an oriented matroid $\mathcal{M}$ is given, the corresponding matroid $\mathcal{M}$ is determined from Definition 6(i). $\underline{\mathcal{M}}$ is the underlying matroid of $\mathcal{M}$ and conversely $\mathcal{M}$ is an orientation of $\mathcal{M}$. A matroid $M$ is called orientable if it has at least one orientation.

Signed circuits are another equivalent axiom systems of oriented matroids. For each circuit $C$ of the underlying matroid, there are two opposite signed circuits $\mathcal{C}^{+}, \mathcal{C}^{-}: C \rightarrow\{-1,1\}$. They are obtained from $\chi$ as follows. Choose elements $x_{1}, \ldots, x_{r}, x_{r+1} \in E_{n}$ such that $C \subseteq\left\{x_{1}, \ldots, x_{r+1}\right\}$ and $\left\{x_{1}, \ldots, x_{r}\right\}$ is a basis of the underlying matroid. Then $\mathcal{C}^{+}\left(x_{i}\right)=(-1)^{i} \chi\left(x_{1}, \ldots, x_{i-1}, x_{i+1}, \ldots, x_{r+1}\right)$ and $\mathcal{C}^{-}$is similar for $-\chi$.

We call two oriented matroids isomorphic when they are equivalent up to two types of operation, relabeling and reorientation. Relabeling is renaming the elements 
Table 5 Number of non-isomorphic oriented matroids (an asterisk means the corresponding value is not determined)

\begin{tabular}{|c|c|c|c|c|c|c|c|c|c|c|c|}
\hline$r \backslash n$ & 0 & 1 & 2 & 3 & 4 & 5 & 6 & 7 & 8 & 9 & 10 \\
\hline 0 & 1 & & & & & & & & & & \\
\hline 1 & & 1 & & & & & & & & & \\
\hline 2 & & & 1 & 1 & 1 & 1 & 1 & 1 & 1 & 1 & 1 \\
\hline 3 & & & & 1 & 2 & 4 & 17 & 143 & 4890 & 461053 & 95052532 \\
\hline 4 & & & & & 1 & 3 & 12 & 206 & 181472 & $*$ & * \\
\hline 5 & & & & & & 1 & 4 & 25 & 6029 & $*$ & $*$ \\
\hline 6 & & & & & & & 1 & 5 & 50 & 508321 & * \\
\hline 7 & & & & & & & & 1 & 6 & 91 & * \\
\hline 8 & & & & & & & & & 1 & 7 & 164 \\
\hline 9 & & & & & & & & & & 1 & 8 \\
\hline 10 & & & & & & & & & & & 1 \\
\hline
\end{tabular}

*Enumeration not completed

of the ground set and reorientation is reversing the signs of the elements in some subset of the ground set. Sets of oriented matroids equivalent up to each operation are called a relabeling class and a reorientation class. Simple oriented matroids are those whose underlying matroid is simple.

The result of isomorph-free enumeration of oriented matroids by Finschi and Fukuda [18-20] is shown in Table 5. In the special case of uniform oriented matroids, Aichholzer and Krasser determined 41,848,591 oriented matroids of rank 3 on eleven elements [2]. Comparing Table 5 to Table 4, we can observe easily that the number of oriented matroids is much larger than that of matroids.

\subsection{Method and Results of Testing Orientability}

In this section, we present a method of testing orientability of matroids and some computational results.

A matroid $M$ of rank $r$ on $E_{n}$ is orientable if and only if there is an alternating map $\chi$ satisfying following conditions: (i) the set $\left\{\left\{x_{1}, \ldots, x_{r}\right\} \mid \chi\left(x_{1}, x_{2}, \ldots, x_{r}\right) \neq 0\right\}$ is equal to the set of bases of $M$, and (ii) $\chi$ satisfies the 3-term Grassman-Plücker relations (see Definition 6). The condition (i) determines whether the value of $\chi$ on each $r$-subset of $E_{n}$ is zero or non-zero. Thus, the remaining problem is whether there is an assignment of -1 and 1 to the values of $\chi$ on $r$-subset corresponding to bases of $M$.

Our method for testing orientability is to translate this problem into an instance of SAT problem and solve it with a general SAT solver. We use MiniSAT [14] in our experiments. The variables correspond to the values of $\chi$ on ordered bases of $M$ and the Boolean values true and false correspond to 1 and -1 , respectively. The constraints are the 3-term Grassman-Plücker relations. This method is similar to that for generation of oriented matroids using SAT by Bremner, Bokowski and Gévay [6] and Schewe [37]. However, our case is special since we need only one variable for 
each $r$-subset because whether the value is zero or non-zero is fixed in a problem of testing orientability. The method of Gugisch [25] is similar to ours in generating the underlying matroids first, but does not encode the orientability question as a SAT instance.

We mention some details of the construction of a SAT instance. First, because the original 3-term Grassman-Plücker relations have redundancy from a viewpoint of computation, we use the following equivalent form:

Proposition 4 [4, Lemma 3.5.4] For an alternating mapping $\chi: E_{n}{ }^{r} \rightarrow\{-1,0,1\}$, the 3-term Grassman-Plücker relations are equivalent to the following properties: for any $x_{3}<\cdots<x_{r}, y_{1}<y_{2}<z_{1}<z_{2} \in E_{n}$, the set

$$
\begin{aligned}
S= & \left\{\chi\left(y_{1}, y_{2}, x_{3}, \ldots, x_{r}\right) \cdot \chi\left(z_{1}, z_{2}, x_{3}, \ldots, x_{r}\right),\right. \\
& \chi\left(y_{1}, z_{1}, x_{3}, \ldots, x_{r}\right) \cdot \chi\left(y_{2}, z_{2}, x_{3}, \ldots, x_{r}\right), \\
& \left.\chi\left(y_{1}, z_{2}, x_{3}, \ldots, x_{r}\right) \cdot \chi\left(y_{2}, z_{1}, x_{3}, \ldots, x_{r}\right)\right\}
\end{aligned}
$$

satisfies $\{-1,1\} \subseteq S$ or $S=\{0\}$.

Next, we add two types of constraint to narrow the search space. Because if $\chi$ is a chirotope then $-\chi$ is too, we may fix the value of $\chi$ on one freely selected ordered base. With this constraint, each orientation of a matroid corresponds to one satisfiable truth assignment of the SAT formula.

In addition, if $\chi$ is a chirotope, its reorientations are too. We may add constraints so that only one selected representative of a reorientation class corresponds to one satisfiable truth assignment of the SAT. Such constraints are as follows. Suppose that a matroid $M$ on $E_{n}$ is connected i.e. for any $e_{i}, e_{j} \in E_{n}$, there is a circuit containing both $e_{i}$ and $e_{j}$. Suppose that $C_{i}$ is a circuit containing both $e_{1}$ and $e_{i}(i \neq 1)$. If $M$ has an orientation and its signed circuit $\mathcal{C}_{i}$ is one of the two opposite signatures of $C_{i}$, there is a reorientation of it such that $e_{1}$ and $e_{i}$ have the same sign in $\mathcal{C}_{i}$. By translating this in terms of chirotopes for each $2 \leq i \leq n$, we obtain $n-1$ constraints. If $M$ is not connected, we proceed similarly for each connected component. In general, if $M$ has $c$ connected components, there are $n-c$ constraints of this type. Note that these constraints to remove redundant reorientations are obtained by utilizing the structure of the underlying matroid.

Since we have formulated a SAT problem where each solution corresponds to a reorientation class of our matroid, we can enumerate reorientation classes by enumerating solutions to the SAT problem. For a feasible SAT problem (i.e. an orientable matroid), each successive solution can typically be found relatively quickly. If the desired output is isomorphism classes, then this technique is only efficient if there are relatively few reorientation classes per isomorphism class. Although in the extreme case of the uniform matroid this ratio is huge, our experiments with simple matroids on nine elements show that the median is 3.33 reorientation classes per isomorphism class, and the third quartile is 8.0. These results suggest that a SAT-based technique for enumerating isomorph-free orientations of a given matroid may be effective for many inputs. For inputs with many reorientation classes, an alternative approach such as that of Finschi and Fukuda [18] or Gugisch [25] may be faster. 
Fig. 13 The Fano matroid $\mathrm{F}_{7}$ and the MacLane matroid $\mathrm{ML}_{8}$
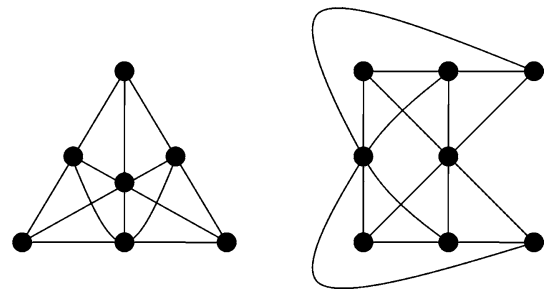

We close this subsection by presenting our experiments on orientability of matroids. Because all matroids on at most six elements are orientable, we have only to investigate matroids on $n \geq 7$ elements. Because all matroids of rank at most 2 are orientable [4, pp. 247-248], we have only to investigate matroids of rank 3 or more. Furthermore, because orientability is an invariant under duality, we have only to consider $r \leq n / 2$. Moreover, because removing loops and parallel elements does not affect orientability, we have only to investigate simple matroids. The results are summarized in Table 2. Because it is hard to determine orientability of all rank 4 matroids on ten elements, we investigate a random sample of 1,000,000 matroids to estimate the ratio of non-orientable ones. We discuss non-orientable matroids further in Sect. 4.3.

\subsection{Non-orientable Matroids}

Non-orientable matroids are of interest both in the study of matroids and of oriented matroids because they show that these two concepts are essentially different, and neither dominates the other. Minimal non-orientable matroids are of particular interest.

In this section, we limit the discussion to rank 3 matroids. As shown in Table 2, we have determined minimal non-orientable matroids on $n \leq 12$ elements. We will now examine more closely the smaller ones. The Fano matroid $F_{7}$ is known as the unique non-orientable matroid on seven elements. $\mathrm{F}_{7}$ is also minimal non-orientable. The MacLane matroid $\mathrm{ML}_{8}$ is a known minimal non-orientable matroid on eight elements. Our result shows $\mathrm{ML}_{8}$ is the unique minimal one on eight elements. $\mathrm{F}_{7}$ and $\mathrm{ML}_{8}$ are shown in Fig. 13. In fact, Ziegler presented the infinite sequence of minimal non-orientable matroids [41], which consists of one matroid on $3 n-1$ elements for each $n \geq 3$ and starts from $\mathrm{ML}_{8}$. However, these are only some of the minimal nonorientable matroids.

As the minimal non-orientable instances following $\mathrm{F}_{7}$ and $\mathrm{ML}_{8}$, we present two minimal non-orientable matroids on nine elements in Fig. 14. These two contains $\mathrm{F}_{7}^{-}$, a single-element deletion of $\mathrm{F}_{7}$, as a submatroid. and may be related to $\mathrm{F}_{7}$. For matroids on 10 or more elements, the number of minimal non-orientable ones grows rapidly. We do not show them individually here but they do not have so symmetric structure as in cases $n \leq 9$.

We also investigate these matroids from a viewpoint of representability. If a field $F$ is $G F(2)$ and $G F(3), M$ is called a binary matroid and a ternary matroid, respectively. $\mathrm{F}_{7}$ is known as the unique non-orientable one among binary rank 3 matroids. We obtained the fact that $\mathrm{ML}_{8}$ is special in the following sense: 
Fig. 14 Minimal non-orientable matroids on nine elements
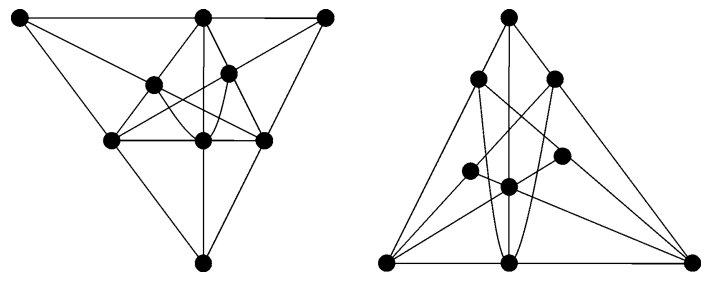

Theorem $8 M L_{8}$ is the unique minimal non-orientable matroid among ternary rank 3 matroids. That is to say, a rank 3 ternary matroid is non-orientable if and only if it contains $M L_{8}$ as a minor.

From these facts, minimal non-orientable matroids of rank 3 are neither binary nor ternary other than $\mathrm{F}_{7}$ and $\mathrm{ML}_{8}$.

In Björner et al. [4, Chap. 6], the authors give six sufficient conditions for nonorientability of matroids. We tested these conditions using our enumeration of rank 3 matroids on at most twelve elements. None of the conditions were very strong in our experiments, with two failing to be satisfied by any non-orientable matroid. Björner et al. also asked whether the six conditions are independent. Our enumeration shows that three of the six conditions (namely the first and third of Proposition 6.6.1, and the third from Proposition 6.6.3) are indeed independent from the others.

\section{Concluding Remarks}

In this paper, we analyze two types of incidence problem in discrete geometry, the points-lines-planes conjecture and the Sylvester-Gallai type problems, using matroids. Matroids are one of abstract settings for combinatorial structure of geometric configurations such as point configurations and hyperplane arrangements. Matroids merely encode incidence information such as collinearity or coplanarity, but their simplicity makes it possible to enumerate a large amount of matroids.

Thus we introduced a new orderly algorithm for the enumeration of matroids, i.e. one that does not require pairwise isomorphism tests. This algorithm is based on a canonical representation for matroids, and generates matroids of a desired rank and number of elements by recursively generating those with one less element, and generating single-element extensions. We introduce the notion of a taboo flat as a way of reducing the number of single-element extensions to be checked.

Our algorithm can enumerate more larger-size matroids than the previous ones. The main computational result is the complete classification of matroids of rank 4 on ten elements. When geometric configurations corresponding to specific matroids, the configurations should be analyzed on oriented matroids, derived from the matroids. We proposed the testing algorithm of matroid orientability by reduction to a boolean satisfiability (SAT) problem. With this technique, we enumerated oriented matroids of rank 3 and at most twelve elements and rank 4 and at most nine elements, and found several new minimal non-orientable matroids. 


\section{References}

1. Aichholzer, O., Aurenhammer, F., Krasser, H.: Enumerating order types for small point sets with applications. Order 19, 265-281 (2002)

2. Aichholzer, O., Krasser, H.: Abstract order type extension and new results on the rectilinear crossing number. Comput. Geom. 36, 2-15 (2006)

3. Betten, A., Betten, D.: Linear spaces with at most 12 points. J. Comb. Des. 7, 119-145 (1999)

4. Björner, A., Las Vergnas, M., Sturmfels, B., White, S.N., Ziegler, G.M. (eds.): Oriented Matroids, 2nd edn. Cambridge University Press, Cambridge (1999)

5. Blackburn, J.E., Crapo, H.H., Higgs, D.A.: A catalogue of combinatorial geometries. Math. Comput. 27, 155-166 (1973)

6. Bremner, D., Bokowski, J., Gévay, G.: Symmetric matroid polytopes and their generation. Eur. J. Comb., in press (2008). http://dx.doi.org/10.1016/j.ejc.2008.12.006

7. Brüngger, A., Marzetta, A., Fukuda, K., Nievergelt, J.: The parallel search bench ZRAM and its applications. Ann. Oper. Res. 90, 45-63 (1999)

8. Crapo, H.H.: Single-element extensions of matroids. J. Res. Natl. Bur. Stand. 69B, 55-65 (1965)

9. Crowe, D.W., McKee, T.A.: Sylvester's problem on collinear points. Math. Mag. 41, 30-34 (1968)

10. Csima, J., Sawyer, E.T.: There exist $6 n / 13$ ordinary points. Discrete Comput. Geom. 9, 187-202 (1993)

11. Davis, M., Logemann, G., Loveland, D.: A machine program for theorem-proving. Commun. ACM 5(7), 394-397 (1962)

12. Davis, M., Putnam, H.: A computing procedure for quantification theory. J. ACM 7(3), 201-215 (1960)

13. Dirac, G.A.: Collinearity properties of sets of points. Q. J. Math. 2, 221-227 (1951)

14. Eén, N., Sörensson, N.: Minisat page. http://minisat.se/

15. Erdös, P.: Problems for solution, \# 4065. Am. Math. Mon. 50, 65 (1943)

16. Erdös, P.: Solution of problem 4065. Am. Math. Mon. 51, 169-171 (1944)

17. Faradzev, I.A.: Generation of nonisomorphic graphs with a given degree sequence. In: Algorithmic Studies in Combinatorics, pp. 11-19. Nauka, Moscow (1978) (Russian)

18. Finschi, L.: A graph theoretical approach for reconstruction and generation of oriented matroids. PhD thesis, Swiss Federal Institute of Technology Zurich (2001)

19. Finschi, L., Fukuda, K.: Homepage of oriented matroids. http://www.om.math.ethz.ch/

20. Finschi, L., Fukuda, K.: Generation of oriented matroids-a graph theoretical approach. Discrete Comput. Geom. 27, 117-136 (2002)

21. Finschi, L., Fukuda, K.: Complete combinatorial generation of small point configurations and hyperplane arrangements. In: Aronov, B., Pach, J. (eds.) The Goodman-Pollack Festschrift, pp. 425-440. Springer, Berlin (2003)

22. Folkman, J., Lawrence, J.: Oriented matroids. J. Comb. Theory, Ser. B 25, 199-236 (1978)

23. Goodman, J.E., O'Rourke, J.: Handbook of Discrete and Computational Geometry. CRC Press Series on Discrete Mathematics and Its Applications, 2nd edn. CRC Press, Boca Raton (2004)

24. Grünbaum, B.: The importance of being straight. In: In Proc. 12th Biannual Intern. Seminar of the Canadian Math. Congress, pp. 243-254. Vancouver, 1969 (1970)

25. Gugisch, R.: A construction of isomorphism classes of oriented matroids. In: Klin, M., Jones, G.A., Jurisic, A., Muzychuk, M., Ponomarenko, I. (eds.) Algorithmic Algebraic Combinatorics and Gröbner Bases, pp. 229-249. Springer, Berlin (2009)

26. Hansen, S.: A generalization of a theorem of Sylvester to the lines determined by a finite point set. Math. Scand. 16, 175-180 (1965)

27. Hansen, S.: Contributions to the Sylvester-Gallai theory. PhD thesis, University of Copenhagen (1981)

28. Mayhew, D., Royle, G.F.: Matroids with nine elements. J. Comb. Theory, Ser. B 98, 415-431 (2008)

29. McKay, B.D.: Isomorph-free exhaustive generation. J. Algorithms 26, 306-324 (1998)

30. Melchior, E.: Uber vielseite der projektiven ebene. Deutsche Math. 5, 13 (1940)

31. Motzkin, T.: The lines and planes connecting the points of a finite set. Trans. Am. Math. Soc. 70, 451-464 (1951)

32. Motzkin, Th.: The lines and planes connecting the points of a finite set. Trans. Am. Math. Soc. 70, 451-464 (1951)

33. Oxley, J.G.: Matroid Theory. Oxford University Press, Oxford (1992)

34. Read, R.C.: Every one a winner or how to avoid isomorphism search when cataloguing combinatorial configurations. Ann. Discrete Math. 2, 107-120 (1978) 
35. Richter-Gebert, J.: Testing orientability for matroids is NP-complete. Adv. Appl. Math. 23(1), 78-90 (1999)

36. Royle, G., Mayhew, D.: Small matroids. http://people.csse.uwa.edu.au/gordon/small-matroids.html

37. Schewe, L.: Satisfiability problems in discrete geometry. Dissertation, TU Darmstadt (2007)

38. Seymour, P.D.: On the points-lines-planes conjecture. J. Comb. Theory, Ser. B 33, 17-26 (1982)

39. Sylvester, J.J.: Mathematical question 11851. Educ. Times 45, 231 (1893)

40. Welsh, D.J.A.: Matroid Theory. Academic Press, New York (1976)

41. Ziegler, G.M.: Some minimal non-orientable matroids of rank three. Geom. Dedic. 38, 365-371 (1991) 